\title{
Interferometry of light propagation in pulsed fields
}

\author{
B. DöBRICH and H. GIES \\ Theoretisch-Physikalisches Institut, Friedrich-Schiller-Universität Jena, \\ Max-Wien-Platz 1, D-07ry3 Jena, Germany
}

PACS 12.20.Fv - Quantum electrodynamics: Experimental tests PACS 14.80.-j-Other particles (including hypothetical)

\begin{abstract}
We investigate the use of ground-based gravitational-wave interferometers for studies of the strong-field domain of QED. Interferometric measurements of phase velocity shifts induced by quantum fluctuations in magnetic fields can become a sensitive probe for nonlinear self-interactions among macroscopic electromagnetic fields. We identify pulsed magnets as a suitable strong-field source, since their pulse frequency can be matched perfectly with the domain of highest sensitivity of gravitational-wave interferometers. If these interferometers reach their future sensitivity goals, not only strong-field QED phenomena can be discovered but also further parameter space of hypothetical hidden-sector particles will be accessible.
\end{abstract}

Introduction. - Charged quantum fluctuations as predicted by quantum electrodynamics (QED) induce nonlinear self-interactions of electromagnetic fields [1]. This fundamental violation of the superposition principle of classical electrodynamics has not yet been observed on the level of macroscopic electromagnetic fields. Even though light-by-light interactions have been verified in ex$\checkmark$ periments involving high-energy photons [2], an investigation of nonlinear interactions of macroscopic fields would + probe QED and its vacuum structure in a large-amplitude regime which is comparatively little explored in quantum field theory. In fact, large-amplitude or strong-field experiments not only give access to unprecedented fundamental tests of QED, but also facilitate a search for hypothetical particles with light masses and weak couplings to photons (hidden-sector searches). This prospect has recently triggered a remarkable growth of experimental and theoretical activities concerned with strong-field and optical set-ups, for recent reviews see $[3,4]$.

A sensitive probe for vacuum nonlinearities is light propagation in strong electromagnetic fields. The lowest-order nonlinear modifications of Maxwell's theory as induced by QED vacuum polarization are described by the (lowestorder) Heisenberg-Euler Lagrangian [1],

$$
\mathcal{L}=\frac{1}{2}\left(\mathbf{E}^{2}-\mathbf{B}^{2}\right)+\frac{2 \alpha^{2}}{45 m^{4}}\left(\mathbf{E}^{2}-\mathbf{B}^{2}\right)^{2}+7 \frac{2 \alpha^{2}}{45 m^{4}}(\mathbf{E} \cdot \mathbf{B})^{2},
$$

where the scale of nonlinearities is set by the electron mass $m, \alpha \simeq 1 / 137$ denotes the fine-structure constant, and we use $\hbar=c=1$. The resulting field equations predict that a plane wave in a magnetic field $\mathbf{B}$ in vacuum propagates at a reduced phase and group velocity $[5,6]$,

$$
v_{i}=1-\frac{a_{i}}{45} \frac{\alpha^{2}}{m^{4}} B^{2} \sin ^{2} \theta, \quad i=\perp, \|, \quad a_{\|}=14, a_{\perp}=8,
$$

where $\theta$ denotes the angle between the $\mathbf{B}$ field and the propagation direction. There are two propagation eigenmodes polarized parallel $\|$ or perpendicular $\perp$ to the plane spanned by $\mathbf{B}$ and the propagation direction. As the numerical coefficients $a_{\|, \perp}$ differ for the two polarization modes, the magnetized quantum vacuum is birefringent. A number of experiments have already been carried out [7-11] or designed $[12,13]$ to look for vacuum birefringence in terms of high-sensitivity polarimetry. The sensitivity limits achieved so far are roughly four orders of magnitude above those necessary for the QED effect.

An alternative to polarimetry is given by absolute phase velocity measurements by interferometry using, e.g., gravitational-wave interferometers, as first suggested by [14], see also [15]. As gravitational-wave interferometers reach their highest sensitivity for optical-path variations at a frequency $f \sim 10^{2} \mathrm{~Hz}$, the requirements for large-scale magnet systems are enormous, as has recently been discussed in detail in a concrete proposal in [16].

Indeed, using gravitational-wave interferometers for the search for nonlinear vacuum phenomena involves many parameters which need to be taken into account in an optimized fashion. In this work, we propose the use of pulsed magnets, as they are developed and used in a sta- 
ble manner in a number of laboratories worldwide at field strengths approaching 100 Tesla. We derive the strain of the optical path induced by repeated magnet pulses, as this is the central observable at gravitational-wave interferometers. We also compute the signal-to-noise ratio for selected interferometers as a criterion for measurability. As we will demonstrate in the following, not only strongfield QED phenomena can be discovered in such set-ups but also further parts of the parameter space of hypothetical hidden-sector particles will be accessible.

QED-induced strain in gravitational-wave interferometers. - Gravitational wave interferometers consist of two perpendicular interferometer arms of equal length $L$. Mirrors at the arms' ends form an evacuated cavity for two in-phase laser beams one for each cavity. An incoming perturbation such as a gravitational wave leads to a relative change $\Delta L$ of the length of the interferometer arms, which manifests itself as a phase difference of the two laser beams. The strain $h(t)=\Delta L / L$ then corresponds to the amplitude of the incoming perturbation. Remarkably, present day interferometers are, in certain frequency ranges, sensitive to a strain of $h \sim 10^{-22}$; future upgrades aim at further improvements of one or two orders of magnitude.

In the context of quantum-induced refractive properties of the vacuum, it is not the arm length but the corresponding optical path length $L$ which can be modified by a strong field. If an external field is applied in a region $x<L$ in one interferometer arm, an optical-path difference $\Delta L$ is induced, $(\Delta L / x)=1-v$. From now on, we concentrate on the parallel mode for which the velocity shift is maximal. For this mode, the resulting strain is

$$
\begin{aligned}
h(t) & =\frac{\Delta L}{L}(t)=\frac{x}{L}(1-v(t))=\frac{x}{L} \frac{a_{\|}}{45} \frac{\alpha^{2}}{m^{4}} B(t)^{2} \\
& \approx \frac{x}{L}\left(9.3 \times 10^{-24}\right)\left(\frac{B(t)}{[1 \mathrm{~T}]}\right)^{2} .
\end{aligned}
$$

For a sizable strain, the magnet-length-to-detector-arm ratio $x / L$ should be maximized. As $x$ is constrained by the technical realizability of the magnetic field coil and the detector sensitivity requires long arm lengths, a suitable compromise between detector arm length and sensitivity has to be found.

Detector sensitivity. - The detector sensitivity to the relative shift of the length of the interferometer arms $\Delta L / L$ is limited by various sources of noise. At low frequencies $f \lesssim 40 \mathrm{~Hz}$, the main limitation arises from seismic activities for ground-based detectors. For instance at $f \sim 10 \mathrm{~Hz}$, which seems ambitious but feasible for big dipole magnets, the sensitivity measure $S_{h}(f)$ (as detailed below) is suppressed by more than three orders of magnitude compared to the peak sensitivity and depletes rapidly for even smaller frequencies. At intermediate and higher frequencies, thermal and shot noise, respectively, limit the detector sensitivity $[17,18]$. We conclude that observing the quantum-induced phenomena requires a magnetic field modulation in the optimal frequency range for a given detector. Pulsed magnets which can be modulated over an appropriate parameter range therefore appear to be good candidates.

Information about the optimal frequency range is encoded in the total spectral density function $S_{h}(f)$ of the noise, see e.g. [18]. For our estimates, we concentrate on advanced LIGO (in preparation) and GEO600 (operational). The projected sensitivity of both detectors slightly depends on the details of the event acting as a source for the interferometric signal. In the case of LIGO, we use the strain sensitivity for neutron-star binaries [19] for our calculations, which is satisfactory over a wide range of intermediate frequencies (for a typical expected sensitivity curve of the advanced LIGO, see [21]). For GEO600, we use the typical sensitivity data available at [20], which can be well approximated by a fit function of the form

$$
S_{h}(f)=S_{0}\left(\left(f_{0} / f\right)^{p_{1}}+2\left(f / f_{0}\right)^{p_{2}}+2\right) / 5,
$$

where $f_{0}=560 \mathrm{~Hz}, S_{0}=7 \times 10^{-44} \mathrm{~Hz}^{-1}, p_{1}=3.8, p_{2}=3$ near the sensitivity maximum. For the advanced LIGO, no simple fit is available. As the signal induced by QED or other hypothetical particles can be predicted exactly, we expect that the interferometer sensitivity can even be optimized for the present phenomenon.

As a measure for the observability of a shift of the optical path $\Delta L / L$, we determine the signal-to-noise ratio (SNR) $d$ of the induced strain. In the present case, the SNR equals the expectation value of the detector output divided by the standard deviation of the output variable due to noise. Using a matched filter (or "Wiener filter") for the signal, the SNR $d$ for a gravitational wave interferometer is given by (see e.g. [18, 22,23] and references therein)

$$
d^{2}=2 \int_{0}^{\infty} \frac{|\tilde{h}(f)|^{2}}{S_{h}(f)} \mathrm{d} f, \quad \tilde{h}(f)=\int_{-\infty}^{\infty} h(t) e^{-2 \pi i f t} \mathrm{~d} t
$$

where $\tilde{h}(f)$ is the Fourier transform of the induced strain.

Pulsed magnetic fields. - As an example, we consider pulsed fields that can be obtained at the Dresden High Magnetic Field Laboratory (HLD) [24]. The HLD aims at providing $100 \mathrm{~T}$ fields generated by a solenoid in a non-destructive set-up, i.e., the infrastructure is maintained and the experiment can in principle be repeated arbitrarily often.

As the magnetic pressure is given by $p_{\text {mag }}=B^{2} / 2 \mu_{0}$, already at fields strengths of about $B=50 \mathrm{~T}$, the pressure on the coils is four orders of magnitude above the atmospheric pressure, which requires a careful coil design. As a consequence, the coils are usually heavily mantled and it is difficult to render the interferometer laser beam orthogonal to the external magnetic field within a single coil setup. In order to maximize the shift of the optical path, a pair of Helmholtz coils has to be used instead. 
In this manner the laser beam of the interferometer can be aligned in parallel to the field coils and thus mainly orthogonal to the magnetic field lines without interfering with the coil mantle.

For such "split coils", a maximum field strength of up to $60 \mathrm{~T}$ appears technically feasible, at a coil diameter of about $x=0.2 \mathrm{~m}$ and a coil separation of $\mathcal{O}(1 \mathrm{~cm})$. As the beam waist of the interferometer lasers is of the order of $\mathrm{cm}$, the interferometer beam can fit between the magnet coils, even though the issue of stray photons may require further discussion which goes beyond the scope of the present work. Also for standard Helmholtz setups, the field is roughly constant at a sizable extent only along the direction of the magnetic field lines, whereas the quantumvacuum effect requires a sizable field length perpendicular to the field lines; the length of the latter is of the order of the coil separation. For the proposed setup, the coil design thus needs to be optimized to provide for high (but not necessarily constant) magnetic field strengths, spatially extending orthogonally to the direction of the field lines.

A typical pulse undergoes a damped oscillation with pulse frequency $\nu_{\mathrm{B}}$ and damping rate $\gamma$. For $N$ subsequent pulses at times $t_{0} \ldots t_{N-1}$, a satisfactory description is given by

$B(t)=B_{0} \sum_{i=0}^{N-1} \theta\left(t-t_{i}\right) \sin \left(2 \pi \nu_{B}\left(t-t_{i}\right)\right) \exp \left(-\gamma\left(t-t_{i}\right)\right)$

Here, we have ignored that successive pulses have no temporal overlap in a single-magnet set-up. However, as the pulse repetition rate $\nu_{\mathrm{P}} \equiv 1 /\left(t_{i+1}-t_{i}\right)$ is much smaller than the damping rate (see below), Eq. (6) is a well justified approximation. The pulse frequency $\nu_{B}$ in Eq. (6) depends on the total capacity of the capacitor banks and can lie in the range $\mathcal{O}(\mathrm{ms} . . \mathrm{s})$, while the damping rate $\gamma$ is mainly determined by the heat capacity of the coil. In addition, the achievable pulse repetition rate $\nu_{\mathrm{P}}$ in a non-destructive mode depends strongly on the desired peak field strength. An ambitious, but nevertheless feasible Helmholtz setup should be able to achieve a maximum field strength of $B_{\max }=60 \mathrm{~T}$, followed by a reverse field of $B_{\min }=-6 \mathrm{~T}$ and thus a damping to about $10 \%$ of the peak field strength ${ }^{1}$. This choice fixes the amplitude of the model pulse (Eq. (6) $B_{0} \approx 148 \mathrm{~T}$ and implies the constraint

$$
\gamma=2 \nu_{B} \ln \left|\frac{B_{\max }}{B_{\min }}\right| .
$$

We use the remaining free parameter $\nu_{B}$ for optimizing the SNR (5) (within the technical limitations). For this, we need the modulus of the Fourier transform of the strain. Ignoring overlap terms of successive pulses as ar-

\footnotetext{
${ }^{1}$ It can be expected that also pulses with damping to about $70 \%$ will be obtainable in the near future. The above pulse parameters are in that sense conservative, since a smaller damping factor $\gamma$ leads to a higher strain (cf. 8)
}

gued above, we find

$$
\begin{aligned}
& |\tilde{h}(f)|^{2}=\frac{x^{2}}{L^{2}}\left(9.3 \times 10^{-24}\right)^{2}\left(\frac{B_{0}}{[1 T]}\right)^{4} \\
& \times \frac{\left(\pi \nu_{B}\right)^{4} \sin ^{2}\left(\pi \frac{f}{\omega_{\mathrm{P}}} N\right) \csc ^{2}\left(\pi \frac{f}{\omega_{\mathrm{P}}}\right)}{\left(\gamma^{2}+\pi^{2} f^{2}\right)\left(\gamma^{4}+\pi^{4}\left(f^{2}-4 \nu_{B}^{2}\right)^{2}+2 \gamma^{2} \pi^{2}\left(f^{2}+4 \nu_{B}^{2}\right)\right)} .
\end{aligned}
$$

For a single pulse $N=1$, the trigonometric functions in Eq. (8) cancel and the dependence on the repetition rate $\nu_{\mathrm{P}}$ drops out, as expected. For a large number of pulses $N$, the trigonometric functions yield a representation of a $\delta$ comb,

$$
\sin ^{2}\left(\pi \frac{f}{\nu_{\mathrm{P}}} N\right) \csc ^{2}\left(\pi \frac{f}{\nu_{\mathrm{P}}}\right) \approx N \sum_{n \in \mathbb{N}} \delta\left(\frac{f}{\nu_{\mathrm{P}}}-n\right) .
$$

At large $N$, only frequencies which are multiples of the pulse repetition rate $\nu_{\mathrm{B}}$ thus contribute to the SNR (5). As $\nu_{\mathrm{B}}$ is much smaller than the frequencies dominating the SNR, the contributing frequencies form a quasi-continuum such that the sum in Eq. (9) can well be approximated by an integral. As a result, the square of the SNR for $N$ pulses can be expressed in terms of the single-pulse result to a good accuracy:

$$
\left.\left.d^{2}\right|_{N} \approx N d^{2}\right|_{1}
$$

The reproducibility of the signal by non-destructive pulsed magnets thus is a lever arm for an enhancement of the SNR by a factor of $\sqrt{N}$. For the advanced LIGO with $L=$ $4000 \mathrm{~m}$, the sensitivity curve has a broad minimum of the order of $S_{h}(f) \approx 10^{-47} \mathrm{~Hz}^{-1}$ for frequencies ranging from approximately $50 \mathrm{~Hz}$ to $500 \mathrm{~Hz}$. Maximizing $d^{2}$ by varying the pulse parameter $\nu_{B}$ yields $\nu_{B} \approx 47 \mathrm{~Hz}$, implying $\gamma \approx$ $217 \mathrm{~Hz}$ by means of Eq. (7). Inserting these values into Eq. (5), we obtain the SNR for a single pulse,

$$
\left.d\right|_{1} ^{\mathrm{LIGO}} \approx 1.9 \times 10^{-2} .
$$

As a result, about $N \approx 2763$ pulses are required in order to achieve a total SNR of $\mathcal{O}(1)$. Depending on the details of the setup, an SNR of $\mathcal{O}(10)$ might eventually be required. For the following feasibility study, however, we only demand for an SNR of $\mathcal{O}(1)$. This is also justified because the expected signal can be predicted to a high accuracy which will allow for an adapted noise filtering.

As mentioned above, the re-cooling time for the magnet system which determines the pulse-repetition rate depends mainly on the pulse energy. A realistic estimate lies in the order of several minutes, implying a continuous operation of the facility for a few days. This appears perfectly feasible. GEO 600 is considerably less sensitive than advanced LIGO but, for our purposes, profits from the shorter arm length of $600 \mathrm{~m}$. Maximizing $d^{2}$ with respect to $\nu_{B}$ yields $\nu_{B} \approx 273 \mathrm{~Hz}$ with $\gamma \approx 1259 \mathrm{~Hz}$ and thus a pulse length below $1 \mathrm{~ms}$. As a result, $N \approx 2.3 \times 10^{6}$ pulses are necessary to observe the QED induced strain at GEO. This corresponds to an unrealistic measurement time of a few years. 
In consequence, GEO in combination with presently available pulsed magnets is not well suited for the observation of the QED induced strain. Nevertheless, it still has a new-physics discovery potential, see below.

Search for hidden-sector particles. - The investigation of vacuum nonlinearities can also be used to search for hypothetical particles with small masses and weak couplings to photons [4]. An apparatus designed to observe the QED effect will also explore a significant range of a new-physics parameter space. Here, we concentrate on potential velocity shifts induced by axion-like particles (ALPs) [25] or minicharged particles (MCPs) [26].

MCPs with charge $Q=\varepsilon e$ and mass $m_{\varepsilon}$ induce vacuum effects analogous to electrons. As the MCP mass can be much lighter than the electron, quantum-induced velocity shifts have to be calculated to all orders in the field strength parameter $\varepsilon e B / m_{\varepsilon}^{2}$ and the frequency $\omega / m_{\varepsilon}$ $[27,28]$. MCP masses corresponding to a Compton wavelength larger than the volume inside the Helmholtz coil cannot be fully resolved within the setup. Thus, the coil separation of $\mathcal{O}(1 \mathrm{~cm})$ constrains the search for MCP masses to $m_{\varepsilon} \gtrsim 2 \times 10^{-5} \mathrm{eV}$. Using the formulas of $[26,28]$ for a Dirac spinor MCP (scalar MCPs behave similarly [28]), we calculate the effect for the polarization component parallel to the external field, which maximizes the velocity shift as in the QED case. For an interferometer laser with $\omega=1.2 \mathrm{eV}$ and the pulse shape as used for the QED effect, we obtain exclusion limits in the fractional charge-mass plane $\left(\varepsilon, m_{\varepsilon}\right)$ by demanding a SNR of $\mathcal{O}(1)$, see Fig 1. These exclusion limits display two characteristic limits which correspond to the two asymptotic limits of the velocity shift: $1-v \sim \varepsilon^{4} B^{2} / m_{\varepsilon}^{4}$ for large masses (cf. Eq. (2)) and $1-v \sim-\varepsilon^{8 / 3} B^{2 / 3} / \omega^{4 / 3}$ for small masses.

We find that already a single-pulse $(N=1)$ measurement at advanced LIGO can approach the current best laboratory bounds [28] derived from PVLAS data [8] with potential minor improvements in the larger-mass range. Assuming a measurement time of ten days at GEO with a magnet re-cooling time of 5 minutes, implying $N \simeq 2880$ pulses would arrive at a similar bound. The small-mass asymptotics of the GEO bounds for $N=1$ and $N=2880$ are also shown in Fig. 1. Advanced LIGO with $N=2763$ as needed for QED can reach a sensitivity of $\varepsilon \simeq 10^{-7}$ for $m_{\varepsilon} \lesssim 0.01 \mathrm{eV}$. This would compete with current cosmological bounds [29]. Of course, astrophysical energy loss considerations can lead to much stronger bounds [30] reaching down to $\varepsilon \sim 10^{-14}$. However, as stellar physics probes a much larger momentum-transfer regime, these bounds depend on the underlying microscopic physics and thus may not apply to laboratory experiments [31]. We conclude that an investigation of the QED effect can also improve current laboratory bounds on MCPs to an unprecedented level.

Axion-like particles (ALPs) are uncharged scalars (S) or pseudo-scalars $(\mathrm{P})$ with a mass $m_{\phi}$ and an effective ALPphoton interaction of the form $\mathcal{L}_{\mathrm{P}}=g \phi_{\mathrm{P}} \mathbf{E} \cdot \mathbf{B}$ or $\mathcal{L}_{\mathrm{S}}=$

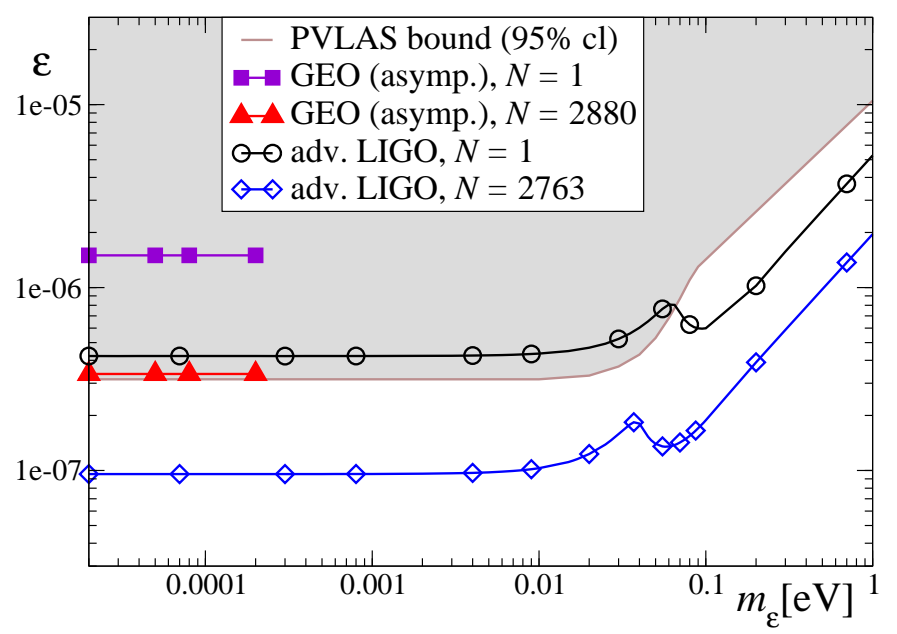

Fig. 1: New-physics discovery potential for spin- $\frac{1}{2}$ minicharged particles (MCP) in the coupling-mass plane. For MCPs, already a single-pulse $(N=1)$ measurement at advanced LIGO (or $N \simeq 2880$ at GEO) can approach or slightly improve the current best laboratory bounds from PVLAS. The use of $N=2763$ pulses as needed for the QED effect can lead to sizable improvements over the whole mass range.

$\frac{1}{2} g \phi_{\mathrm{S}}\left(\mathbf{B}^{2}-\mathbf{E}^{2}\right)$ with coupling $g$. The interaction structure implies that scalars only couple to the $\perp$ mode, whereas pseudo-scalars couple to the $\|$ mode of the propagating light in a magnetic field. Loosely speaking, an effective reduction of the phase velocity arises from the fact that the corresponding photon partly propagates as a massive ALP component. The corresponding velocity shifts read [25]

$$
1-v_{\|}^{\mathrm{P}}=1-v_{\perp}^{\mathrm{S}}=\frac{B^{2}}{2 m_{\phi}^{2}} g^{2}\left(1-\frac{\sin (2 y)}{2 y}\right), \quad y=\frac{x m_{\phi}^{2}}{4 \omega},
$$

where $\omega$ denotes the laser frequency, and $x$ is the length of the magnet interaction region. As discussed in detail in [28], the polarimetry of strong-field particle searches can distinguish between the various particle scenarios, see also [16]. For the current study, we only concentrate on the velocity shift irrespective of the polarization dependence for simplicity.

Let us first consider the ALP parameter range for the coupling $g$ and mass $m_{\phi}$ that can be probed by a single pulse. Using the pulse shape as for the QED case, we obtain the accessible region in the mass-coupling plane, see Fig. 2 for a comparison with the current best laboratory limits from PVLAS and GammeV [32] (see also the BMV experiment [33]). Already a single-pulse measurement at advanced LIGO can improve on existing bounds for masses above $1 \mathrm{meV}$. For $m_{\phi} \gtrsim 4 \times 10^{-4} \mathrm{eV}$, higher number of pulses as needed for the QED effect give access to a parameter space which is significantly larger than current laboratory limits. Again, astrophysical considerations and observations lead to stronger bounds by a few orders of magnitude in the $g \sim 10^{-11} \mathrm{GeV}^{-1}$ regime [34], but may 


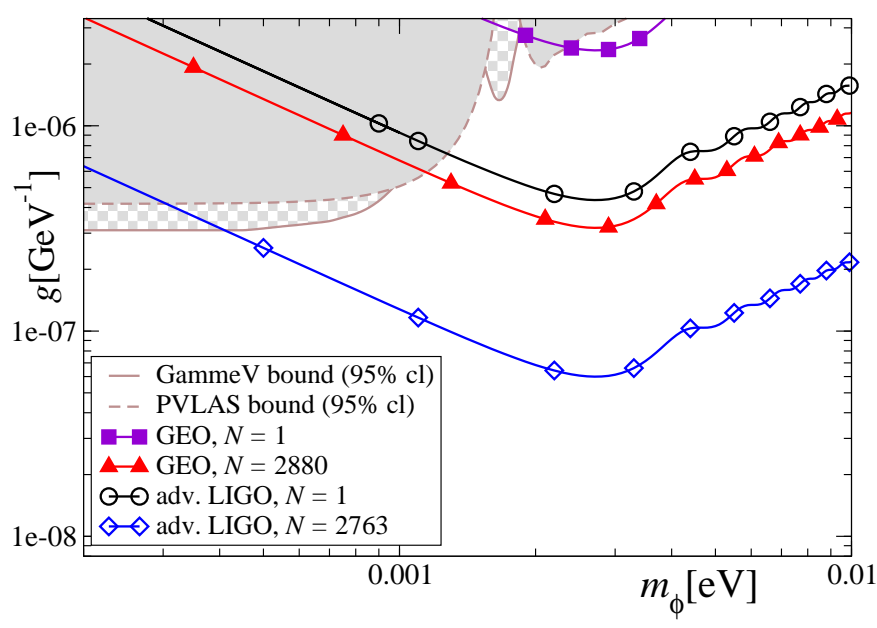

Fig. 2: This figure shows the discovery potential in the coupling-mass plane for axion-like particles (ALP) at LIGO and GEO, respectively. For ALPs, the current best laboratory bounds by GammeV and PVLAS can be improved significantly in the meV mass range and above by advanced LIGO as well as already by GEO.

not be directly applicable to laboratory experiments due to a different momentum-transfer regime [31].

Conclusion. - The sensitivity goal of ground-based gravitational-wave interferometers appears well suited to use these experiments for exploring the strong-field domain of QED. In order to generate a strongly magnetized quantum vacuum in such an interferometric experiment, we have identified pulsed magnets as an advantageous strong-field source for two reasons: they provide extremely strong laboratory magnetic fields, and their pulse frequency can be perfectly matched with the region of highest sensitivity of the gravitational-wave interferometers.

For our quantitative estimates, we have concentrated on the advanced LIGO detector, as its sensitivity goal matches with currently available field strengths already in a rather conservative estimate. Pushing the various components to their limits may facilitate a detection also at the gravitational-wave interferometers, which are currently operational such as GEO 600. Also the fact, that the quantum-induced signal can be theoretically predicted to a good accuracy may give rise to an improved noise filtering.

From a general perspective, the QED velocity shift as well as the MCP signal in the large-mass domain and the dispersive ALP effect scale with $x B^{2}$, where $B$ is the amplitude and $x$ the extent of the magnetic field. For the use of gravitational-wave interferometers, also a suitable time variation of the magnetic field is needed. Whereas pulsed fields profit from extremely high fields and a suitable time variation, their deficit is a smaller extent in comparison to dipole magnets. Since pulsed fields win roughly an or- der of magnitude in the field strength and lose an order of magnitude in the field extent, the quantity $x B^{2}$ can generically still be an order of magnitude larger for pulsed fields than for dipoles. A similar consideration has inspired the development and use of pulsed magnets in the BMV experiment $[10,33]$ which finally aims at a parameter goal of $x B^{2} \simeq 600 \mathrm{~T}^{2} \mathrm{~m}$ (recent experimental results of $\mathrm{BMV}$ have been achieved with $x B^{2} \simeq 40 \mathrm{~T}^{2} \mathrm{~m}$ ) [10]. The pulsed Helmholtz coil configuration considered in this work which is inspired by ongoing experiments at the Dresden High Magnetic Field Laboratory would yield $x B^{2} \simeq 720 \mathrm{~T}^{2} \mathrm{~m}$, which agrees with the design goal of BMV. But whereas the time dependence of the field pulse at BMV can be a disturbing factor for the BMV polarimetry, the time structure of the field pulse is a necessary and advantageous key feature for a strong-field experiment at a gravitationalwave interferometer.

Given the prospect of exploring a new parameter regime of strong-field quantum field theory with implications for the search for new elementary particles, establishing a strong-field quantum-vacuum program at gravitationalwave interferometers appears to be worthwhile.

$$
* * *
$$

We would like to thank Achamveedu Gopakumar, Thomas Herrmannsdörfer, Jörg Jäckel, Guiseppe Ruoso, Gerhard Schäfer and David Shoemaker for helpful discussion. This work is supported by DFG SFB-TR18 and under contract Gi 328/5-1 (Heisenberg program).

\section{REFERENCES}

[1] W. Heisenberg and H. Euler, Z. Phys. 98 (1936) 714; V. Weisskopf, Kong. Dans. Vid. Selsk. Math-fys. Medd. XIV, 166 (1936); J. S. Schwinger, Phys. Rev. 82, 664 (1951).

[2] S. Z. Akhmadaliev et al., Phys. Rev. C 58, 2844 (1998); Phys. Rev. Lett. 89, 061802 (2002); D. L. Burke et al., Phys. Rev. Lett. 79, 1626 (1997).

[3] M. Marklund and J. Lundin, arXiv:0812.3087, to appear in Eur. Phys. J. D, (2009); G. V. Dunne, arXiv:0812.3163, to appear in Eur. Phys. J. D, (2009).

[4] H. Gies, arXiv:0812.0668, to appear in Eur. Phys. J. D, (2009); J. Phys. A 41, 164039 (2008).

[5] R. Baier and P. Breitenlohner, Act. Phys. Austriaca 25, 212 (1967); Nuov. Cim. B 47117 (1967); S. L. Adler, Annals Phys. 67, 599 (1971).

[6] W. Dittrich and H. Gies, Springer Tracts Mod. Phys. 166, 1 (2000).

[7] R. Cameron et al., Phys. Rev. D 47 (1993) 3707.

[8] E. Zavattini et al., Phys. Rev. Lett. 96 (2006) 110406; Phys. Rev. D 77, 032006 (2008).

[9] M. Bregant et al. [PVLAS Collaboration], Phys. Rev. D 78, 032006 (2008) arXiv:0805.3036 [hep-ex]].

[10] R. Battesti et al. , Eur. Phys. J. D 46, 323 (2008).

[11] S. J. Chen, H. H. Mei and W. T. Ni, Mod. Phys. Lett. A 22, 2815 (2007).

[12] P. Pugnat et al., CERN-SPSC-2006-035. 
[13] T. Heinzl, B. Liesfeld, K. U. Amthor, H. Schwoerer, R. Sauerbrey and A. Wipf, Opt. Commun. 267, 318 (2006).

[14] D. Boer and J. W. Van Holten, arXiv:hep-ph/0204207.

[15] V. I. Denisov, I. V. Krivchenkov and N. V. Kravtsov, Phys. Rev. D 69, 066008 (2004).

[16] G. Zavattini and E. Calloni, arXiv:0812.0345 [physics.insdet].

[17] P. R. Saulson, Fundamentals of interferometric gravitational wave detectors, Singapore, World Scientific (1994), K. S. Thorne, arXiv:gr-qc/9506086

[18] D. G. Blair, The Detection Of Gravitational Waves, Cambridge UP (1991).

[19] D. Shoemaker, private communication

[20] http://www.geo600.uni-hannover.de/geocurves/

[21] J. R. Smith [LIGO Scientific Collaboration], arXiv:0902.0381 [gr-qc].

[22] B. F. Schutz, in Proc. Les Houches School on Astrophysical Sources of Gravitational Radiation, Cambridge UP (1995).

[23] K.S. Thorne, in Three hundred years of gravitation, S.W. Hawking, W. Israel (ed.), 330 (1987).

[24] J. Wosnitza, et al., in 2006 IEEE International Conference on Megagauss Magnetic Field Generation and Related Topics, G.F. Kiuttu, et al. (ed.) 197 (2008); J. Wosnitza, et al. Volume 1003, pp. 311-315 AIP Conference Proceedings (2008); J. Wosnitza, et al., J. Magn. Magn. Mat. 310, 2728 (2007).

[25] L. Maiani, R. Petronzio and E. Zavattini, Phys. Lett. B 175, 359 (1986); G. Raffelt and L. Stodolsky, Phys. Rev. D 37, 1237 (1988).

[26] H. Gies, J. Jaeckel and A. Ringwald, Phys. Rev. Lett. 97, 140402 (2006).

[27] W. y. Tsai and T. Erber, Phys. Rev. D 12, 1132, (1975).

[28] M. Ahlers, H. Gies, J. Jaeckel and A. Ringwald, Phys. Rev. D 75, 035011 (2007).

[29] A. Melchiorri, A. Polosa and A. Strumia, Phys. Lett. B 650, 416 (2007).

[30] S. Davidson, S. Hannestad and G. Raffelt, JHEP 0005, $003(2000)$

[31] J. Jaeckel, E. Masso, J. Redondo, A. Ringwald and F. Takahashi, Phys. Rev. D 75, 013004 (2007).

[32] A. S. Chou et al., Phys. Rev. Lett. 100, 080402 (2008).

[33] C. Robilliard et al., Phys. Rev. Lett. 99, 190403 (2007).

[34] K. Zioutas et al., Phys. Rev. Lett. 94 (2005) 121301. 